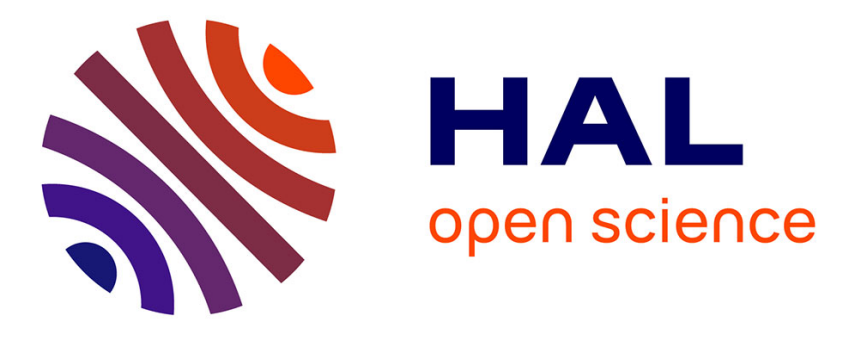

\title{
Liquid Crystal Films as Active Substrates for Nanoparticle Control
}

\author{
Ines Gharbi, Viviana Palacio-Betancur, Habib Ayeb, Dominique Demaille, \\ Juan J de Pablo, Randall D Kamien, Emmanuelle Lacaze
}

\section{- To cite this version:}

Ines Gharbi, Viviana Palacio-Betancur, Habib Ayeb, Dominique Demaille, Juan J de Pablo, et al.. Liquid Crystal Films as Active Substrates for Nanoparticle Control. ACS Applied Nano Materials, 2021, 4 (7), pp.6700-6708. 10.1021/acsanm.1c00680 . hal-03356166

\section{HAL Id: hal-03356166 https://hal.sorbonne-universite.fr/hal-03356166}

Submitted on 27 Sep 2021

HAL is a multi-disciplinary open access archive for the deposit and dissemination of scientific research documents, whether they are published or not. The documents may come from teaching and research institutions in France or abroad, or from public or private research centers.
L'archive ouverte pluridisciplinaire HAL, est destinée au dépôt et à la diffusion de documents scientifiques de niveau recherche, publiés ou non, émanant des établissements d'enseignement et de recherche français ou étrangers, des laboratoires publics ou privés. 


\section{Liquid Crystal Films as Active Substrates for}

\section{Nanoparticle Control}

Ines Gharbi, ${ }^{1,2}$ Viviana Palacio-Betancur, ${ }^{3}$ Habib Ayeb,${ }^{2}$ Dominique Demaille, ${ }^{1}$ Juan J. de Pablo, ${ }^{3,4}$ Randall D. Kamien ${ }^{5}$ and Emmanuelle Lacaze ${ }^{* 1}$

${ }^{1}$ Sorbonne Université, CNRS, Institut des Nanosciences de Paris, INSP, F-75005 Paris, France

${ }^{2}$ Université de Tunis El Manar, Faculté des Sciences de Tunis, LR99ES16 Physique de la Matière Molle et de la Modélisation Electromagnétique, 2092, Tunis, Tunisie

${ }^{3}$ Pritzker School of for Molecular Engineering, University of Chicago, Chicago, IL 60637, USA

${ }^{4}$ Materials Science Division, Argonne National Laboratory, Lemont, IL 60439, USA

${ }^{5}$ Department of Physics and Astronomy, University of Pennsylvania, 209 South 33rd Street, Philadelphia, PA 19104, USA 


\section{ABSTRACT:}

Organizing nanoparticles in a controlled way allows us to monitor their optical properties, in particular if it concerns metallic nanoparticles. It is particularly interesting to organize them on top of liquid crystal films to take advantage, in a second step, of the easy actuation of liquid crystals with external parameters like temperature, electric fields, etc... We show that despite their fluidity, nematic and smectic films allow formation of well-ordered hexagonal domains of gold spherical nanoparticles (AuNPs) at their surface but we also show that both nematic films and AuNP domains impact each other. Using Optical Microscopy, Atomic Force Microscopy (AFM), Scanning Electron microscopy (SEM), and Spectrophotometry, we compare nematic, polymer stabilized nematic and smectic films with AuNP domains made of NPs of diameter $6 \mathrm{~nm}$. On The liquid crystal films depressions are revealed below the AuNP domains whereas the AuNP domains appear well-organized but with a hexagonal period shortened with respect to AuNP monolayers formed on hard substrates. We interpret these features by the anchoring tilt imposed by the AuNP domains on the molecules. The smectic-A layers characteristic of the nematic surface transform into smectic-C layers which induce formation of depression. The energy penalty associated with the local smectic-A/smectic-C transition induces the shortening of the AuNP domain period in order to decrease the AuNP domain surface. The observed large depth of the polymer stabilized nematic depressions below AuNP domains may be explained either by an increased size of the polymer stabilized smectic layers close to the surface or by an increased number of polymer stabilized smectic liquid crystal smectic layers close to the surface with respect to pure nematic films. 


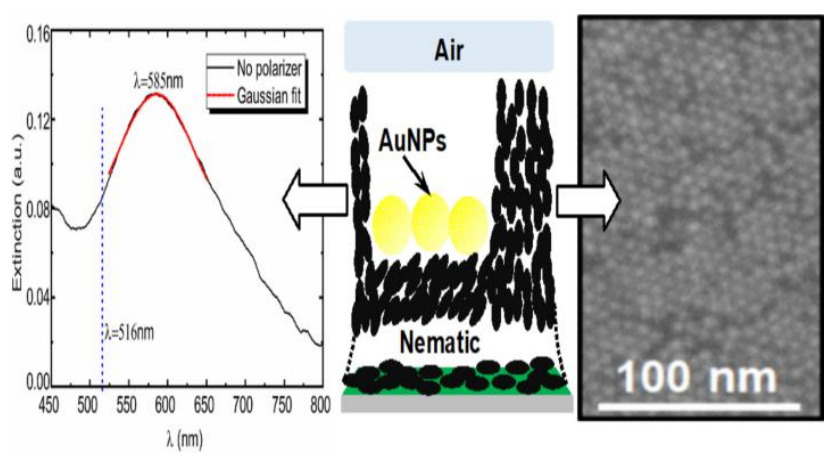

KEYWORDS: liquid crystal, gold nanoparticle, nanoparticle control, plasmon extinction, spectrophotometry, AFM, SEM

Liquid crystals (LCs) are the original nanomaterials: subjugated by surface forces, electromagnetic fields, and flow they can be organized, actuated, and switched while maintaining sub-micron order. When combined with nanoparticles (NPs), these composites become of interest for applications ${ }^{1,2}$ either to induce a modification of LC properties in presence of NPs, ${ }^{3-7}$ or to allow for controlled NP organizations mediated by the LC matrix. These last applications rely on the anisotropy of the LC matrix and its ease of manipulation through external stimuli (temperature, electric field) to build NP clusters that can be modified on demand by these external stimuli. ${ }^{8}$ Commonly, NPs aggregate in a LC bulk without a particular ordering. ${ }^{9,10}$ One alternative to engineer ordered NP structures is using topological defects, regions with mismatching LC molecular orientation, as sites for adsorbing NPs. ${ }^{11-15}$ As a result, topological defects can be used as templates for the formation of NP clusters with supramolecular order. ${ }^{16-19}$

However, forming NP-LC composites through defect engineering is a challenging task since not only does it require the formation of well-defined topological defect arrays, but also it is limited to NP sizes small enough to match the topological defect core sizes and surface chemistries that stabilize the defect morphology of interest. Efforts to use a LC matrix with a low density of defects 
as a substrate for NP self-assembly at the LC surface without the formation of uncontrolled clusters is therefore another interesting challenge. It has been reported that a cholesteric LC film, with a molecular pitch of the order of microns, exhibits a fingerprint texture which guides the NPs into ribbon-like assemblies, revealing the presence of more favourable areas at the surface of these modulated LC matrix. ${ }^{20-24}$ However a deep study of the local NP structure that drives the NP optical properties, in particular the light absorption when plasmonic NPs are concerned, and its relationship with the LC matrix specificity is still lacking.

In this work, we demonstrate the stabilization of large collections of gold nanospheres (AuNP) domains on top of defect-free $5 \mathrm{CB}$ nematic and polymer stabilized 5CB nematic films. These domains exhibit hexagonal packing, similar to ordered domains of particles on hard substrates. The presence of NP domains alters the topography of the free-standing $5 \mathrm{CB}$ film, while the interAuNP distance between AuNPs is shortened and thus red-shifts the plasmonic wavelength associated with AuNP light absorption. We show that similar phenomena are induced at the surface of free-standing $8 \mathrm{CB}$ smectic-A films and interpret these mutual modifications as arising from the AuNP-induced variation of anchoring at the interface.

\section{RESULTS}

Optical Microscopy Observations. 5CB Nematic films (see "Materials and Methods" - Figure 7a) and polymer stabilized 5CB nematic films (see "Materials and Methods" - Figure 7b-c) on rubbed polyvinyl alcohol (PVA) substrates were prepared according to "Materials and Methods" section - Figure 7. We will call in the following the polymer stabilized 5CB nematic films by PSNLC for Polymer Stabilized Nematic Liquid Crystal ("Materials and Methods" - Figure 7c) and the 5CB nematic films by NLC for Nematic Liquid Crystal. The nematic texture of PSNLC and of NLC, with and without gold spherical nanoparticles (AuNPs - see "Materials and Methods" - 
Figure 7d), was first investigated by optical microscopy between crossed polarizers (POM) in transmission. The textures of the NLC and of the PSNLC appear similar (comparison between Figure $1 \mathrm{a}$ and $1 \mathrm{~b}$ and between Figure $1 \mathrm{~b}$ and SI1). For stability purposes, the results presented here are from PSNLC films unless indicated otherwise (Figure 1).

Profilometer measurements show that the thinnest section of the film is at the border, with a thickness of $6 \mu \mathrm{m}$, and the thickest region is at the center, with a thickness of $18 \mu \mathrm{m}$. These values correspond to the color of the optical microscopy pictures between crossed polarizers observed in Figure 1a for NLC film, assuming that a hybrid film (planar anchoring on the rubbed PVA substrate and homeotropic anchoring at air) of LC has an effective birefringence of $\Delta \bar{n}_{L C}=n_{e f f}$ $n_{0}$ with $\mathrm{n}_{\mathrm{o}}$, the ordinary LC index and $n_{e f f}$, the effective extraordinary index determined by the following equation ${ }^{25}$.

$$
n_{e f f}=\frac{1}{e} \int_{0}^{e} \frac{n_{o} n_{e}}{\sqrt{n_{e}^{2} \cos ^{2} \theta(z)+n_{o}^{2} \sin ^{2} \theta(z)}} d z
$$

with e the overall thickness, $\theta(\mathrm{z})$ the molecular orientation throughout the LC film (the director profile), $\mathrm{n}_{\mathrm{e}}$ and $\mathrm{n}_{\mathrm{o}}$ the extraordinary and ordinary $5 \mathrm{CB}$ indexes.

For homeotropic anchoring at air, using $\mathrm{n}_{\mathrm{o}}=1.537$ and $\mathrm{n}_{\mathrm{e}}=1.735$, it can be calculated that $\Delta \bar{n}_{L C}$ is of the order of $0.1 .^{26}$ It decreased by 0.09 with respect to the nominal $5 \mathrm{CB}$ birefringence $\left(\mathrm{n}_{\mathrm{e}}-\mathrm{n}_{\mathrm{o}}\right)=0.19$. The birefringence of monomer RM257 (with $\mathrm{n}_{\mathrm{o}}=1.508$ and $\left.\mathrm{n}_{\mathrm{e}}=1.687\right)^{27}$ and that of $5 \mathrm{CB}$ are of the same order and we don't expect the polymer birefringence to significantly modify the PSNLC one. 
On top of NLC, droplets of gold spherical nanoparticles (AuNPs of diameter $6 \mathrm{~nm}$ and covered by dodecanethiol ligands - see "Materials and Methods" - Figure 7d) in toluene have been deposited. For PSNLC with AuNPs the polymerization occurred after AuNP deposition. Large filaments or cluster structures are observed as shown in Figure 1b (for AuNP structures on NLC film, see Figure S1). The difference between figures $1 \mathrm{a}$ and $1 \mathrm{~b}$ suggests that these domains are composed of AuNPs and the change in color indicates that their presence modifies the structure of the LC film underneath.

Figures 1c-d show a magnified area of the PSNLC-AuNP polymerized films, where the areas with NPs appear blue-shifted. For example, the free nematic in red changes to yellow, and the free nematic in yellow changes to blue. This blue-shift is systematic but can be more or less visible depending on the LC thickness and most probably also on the size and density of the NP domains. We might attribute this blue-shift to two main phenomena: the domains of AuNPs form depressed regions on the PSNLC film altering its thickness, or the AuNPs impose a change of birefringence in the film. The observation of a large and well-visible blue shift of POM colors in the presence of AuNPs would imply non-negligible thickness changes with respect to the overall film thickness such that the second phenomenon is more likely to occur. In order to study the localization and organization of AuNPs in the domains identified by POM and understand the origin of the local birefringence changes, we have studied PSNLC films with AuNPs using Atomic Force Microscopy (AFM). 
(a)

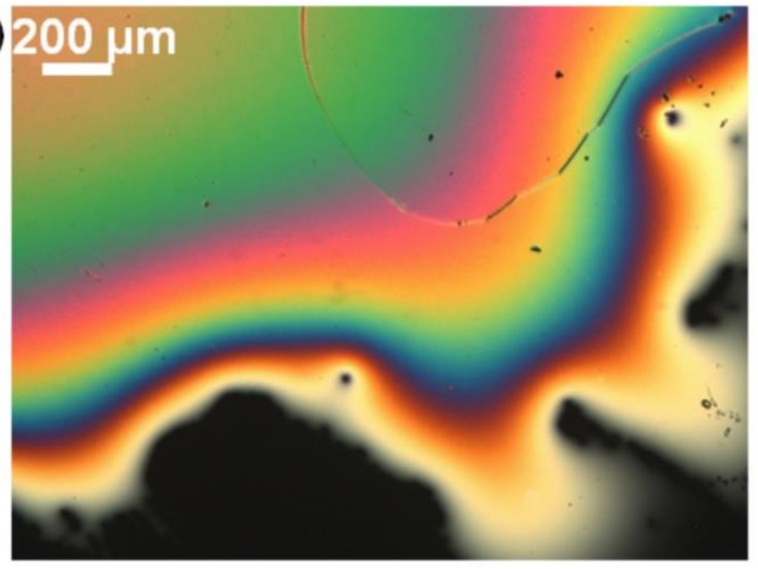

(c)

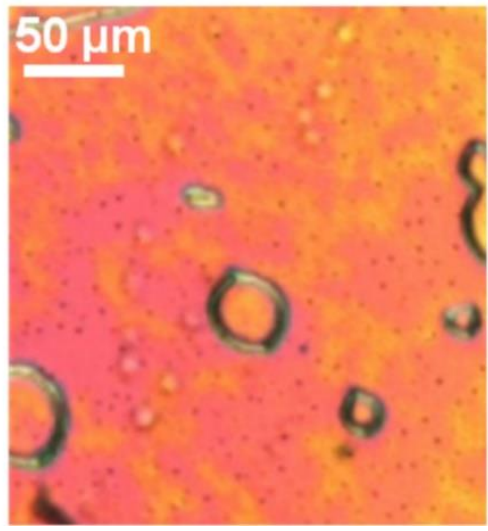

(b)

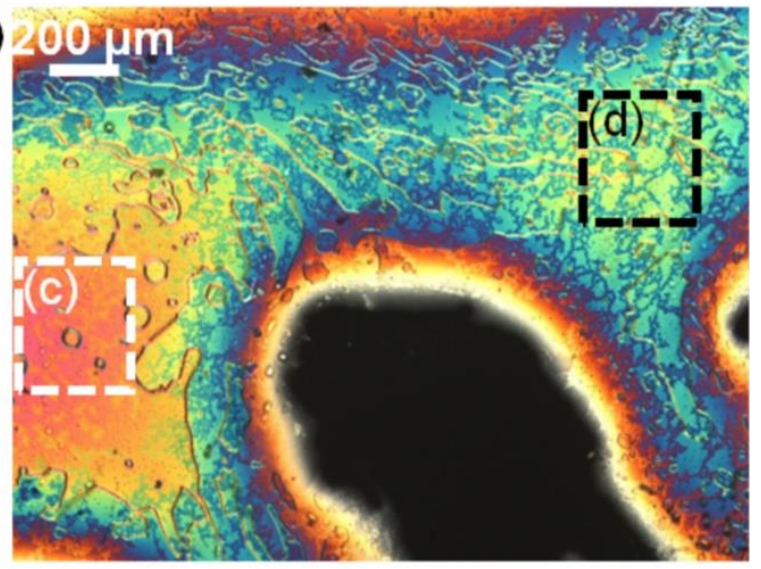

(d)

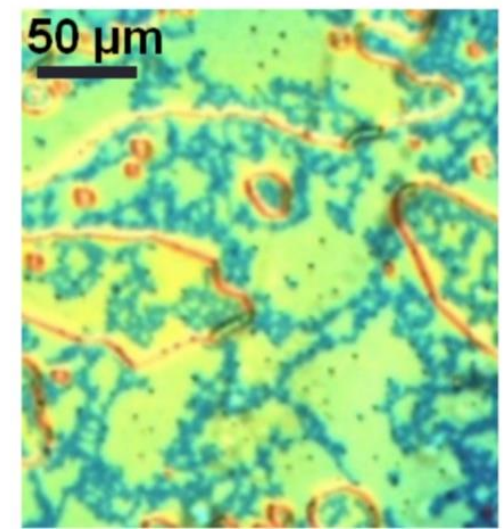

Figure 1. POM images in transmission between crossed polarizers where the molecules are aligned at $45^{\circ}$ of the polarizer: (a) NLC film showing a color variation that correspond to a thickness variation from the edge to the center of the film from 6 to $18 \mu \mathrm{m}$. (b) PSNLC film in the presence of AuNPs forming colored filament-like structures. (c) Enlarged view of AuNPs yellow structures on a red nematic area. (d) Enlarged view of AuNPs blue structures on a yellow nematic area.

AFM Measurements. Figure 2 shows that the macroscopic filament-like shape of the AuNP domains shown in Figure 1 is recovered on the AFM image through a contrast associated with variations in height (Figure 2a) and variations in phase (Figure 2b). AFM results reveal that, more locally, the filaments are divided into islands which are precisely visualized in Figure 2c. The comparison between POM and AFM pictures allows us to identify the islands as associated with 
the AuNP domains. The topography within the AuNP domains and on the free LC film is uniform as shown in Figure 2c-d. The AFM phase variation between the two regions, see Figure 2b, is related to different AFM tip/surface interactions. The observation of such an AFM phase variation demonstrates that the islands are made of AuNP domains localized at the LC surface and not covered in nematic material. It is however not possible to identify the nature of the AuNP packing within the domains given the limited AFM resolution. The change in thickness between the two zones, see Figure 2c-d, shows that the presence of AuNPs organized in domains impacts the nematic structure through the formation of depressions on the PSNLC film surface.
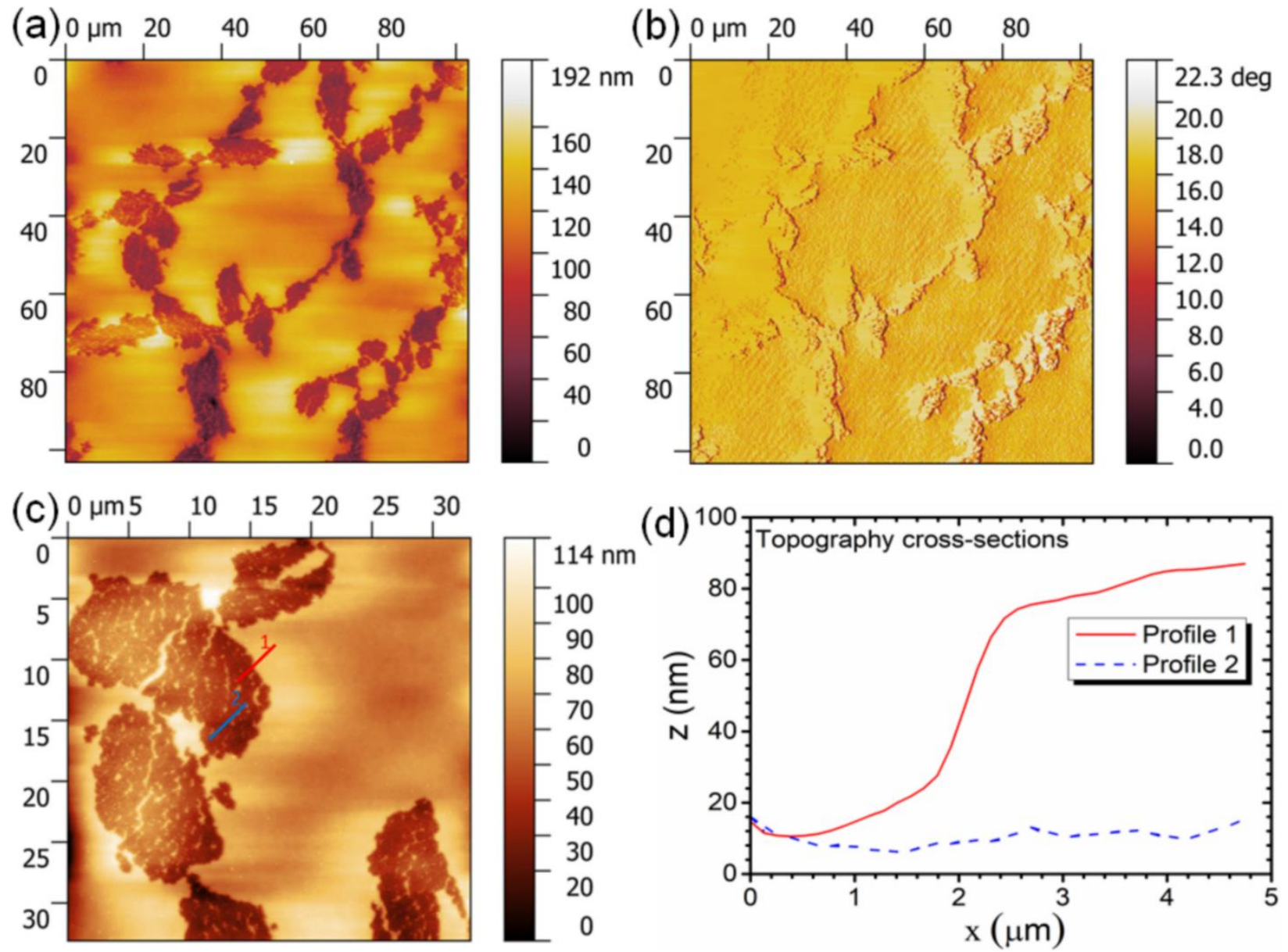

Figure 2. (a) AFM topographical and (b) phase data corresponding to the surface of a PSNLC film in presence of AuNP domains. AuNP regions are visible in the phase data by 
the variations of phase contrast associated with variations of AFM tip interactions with the surface, which coincide with the zones of holes observed in (a). (c) Enlarged topographical view on the domains formed by AuNPs, revealing the formation of well-defined depressions at the nematic surface in presence of AuNPs. (d) Topographical cross-sections according to the lines drawn on (c). The depth of the hole formed by the AuNP domains is $60 \mathrm{~nm}$ with respect to the nematic surface. The bottom of the depression is flat.

The depth of the islands relative to the PSNLC/Air interface, $\triangle \mathrm{e}_{\mathrm{N}-\mathrm{Au}}$, has been determined through 102 measurements performed on three samples. It varies between 20 and $100 \mathrm{~nm}$ with an average value $\Delta \mathrm{e}_{\mathrm{N}-\mathrm{Au}}=82 \pm 24 \mathrm{~nm}$. This $\Delta \mathrm{e}_{\mathrm{N}-\mathrm{Au}}$ value is, indeed, negligible compared to the thickness of the film (between 6 and $18 \mu \mathrm{m}$ ) which confirms that the color variation in Figure $1 \mathrm{~b}-\mathrm{d}$ is caused by a change in the birefringence. This birefringence change must be caused by the AuNP domains replacing air at the nematic surface. A layer of AuNPs of diameter 10nm cannot significantly alter the birefringence of a nematic film of overall thickness between 6 and $18 \mu \mathrm{m}$. This increase of birefringence suggests that the antagonistic boundary conditions of a hybrid film are altered by the presence of the AuNP domains. It is no more perfectly homeotropic, as it was the case in the PSNLC/Air interface, but becomes tilted.

The birefringence change can be estimated according to the following formula:

$$
\Delta \bar{n}=k \frac{\Delta \lambda}{e}
$$

where $\Delta \bar{n}$ is the variation of birefringence with respect to $\Delta \bar{n}_{L C}, \mathrm{k}$ is the optical order, $\Delta \lambda$ is the difference in wavelength between the zones with and without AuNPs identified in the POM pictures and $\mathrm{e}$ is the overall thickness. For the region between yellow and blue (Figure 1d), $\Delta \lambda$ of the order of $100 \mathrm{~nm}$ and $\mathrm{k}=2$. Solving formula (2) for $\Delta \bar{n}$, the change in birefringence should be 
about 0.02 . We calculate $n_{e f f}$ as a function of the molecular orientation throughout the LC film. Firstly, we assume that the director profile (the average LC molecule orientation) follows the analytical solution for a hybrid film described in eq. (1). In such a case a numerical solution for eq. (1) yields an angle $\theta=17^{\circ}$ if $\Delta n$ is increased by 0.02 with respect to homeotropic anchoring at the surface. Secondly, if we assume that the texture in the areas with nanoparticles matches the adjacent texture in the areas without nanoparticles from the PVA $\left(\theta=90^{\circ}\right)$ up until some tilt angle $\theta_{2}$ and then remains at that constant angle all the way to the AuNP interfaces, we find that $\theta_{2}=39^{\circ}$ for an increase $\Delta n$ of 0.02 . This appears to be the maximum LC tilt below the AuNP domains consistent with the observed birefringence. Such tilted anchoring appears consistent with previous results of the literature, since the dodecanethiol ligands grafted on an Au surface can induce tilted anchoring for short enough alkyl chains. ${ }^{28}$
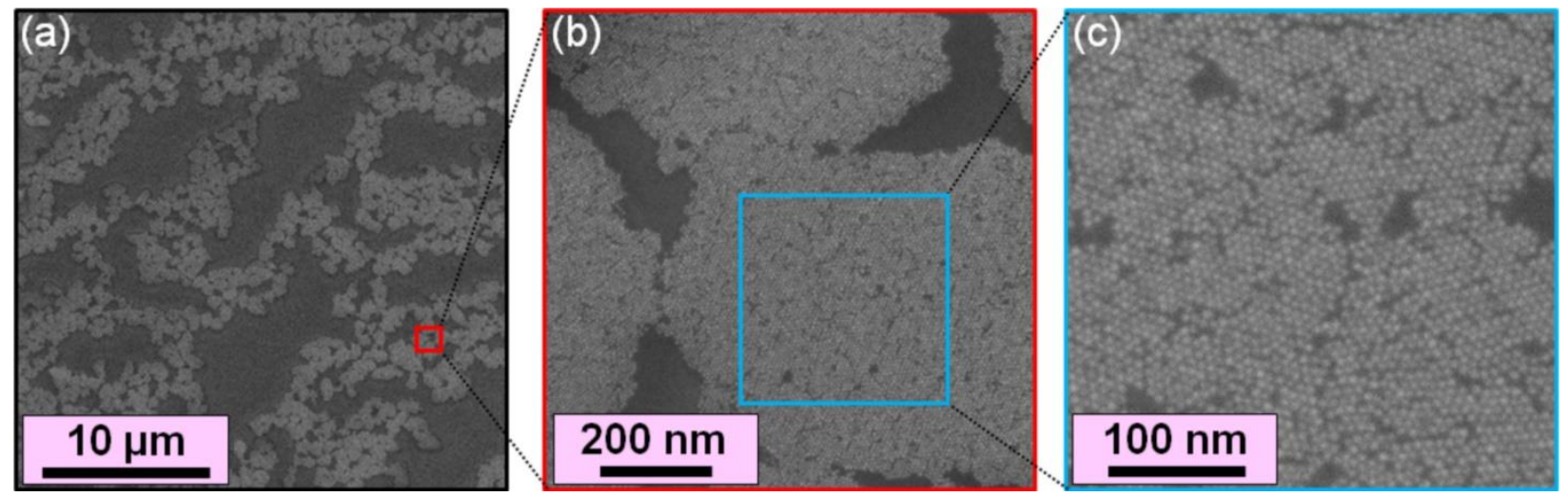

Figure 3. SEM images of the PSNLC-AuNPs sample prepared in the same conditions as the one for spectrophotometry measurements and deposited on a conducting ITO substrate covered by rubbed PVA (a) Large scale picture where AuNP domains appear as large AuNP filaments (b) Enlarged view of the structure showing associations of several domains which correspond to alternating areas with and without AuNP, already seen by AFM. (c) Enlarged 


\section{view inside the AuNP domain revealing an organization of AuNPs into well-ordered hexagonal monolayers.}

Scanning Electron Microscopy (SEM) Investigations. POM and AFM pictures show how AuNPs impact a nematic film, once deposited at the surface. A change of anchoring is induced below the AuNP domains that form at the LC surface. A depression is imposed at the nematic surface for each AuNP domain of depth varying between 20 and $100 \mathrm{~nm}$. We can now ask the reverse question: if and how the AuNPs themselves are affected by the presence of the nematic film below. We have thus studied PSNLC films with AuNPs by SEM (Figure 3) on a slightly more concentrated area than the one studied by AFM. The AuNP domains (in white on Figure 3) are indeed more interconnected than on Figure 2. In contrast with AFM measurements, a zoom on the islands (from Figure 3a to Figure 3c) easily displays the AuNP structure associated with formation of hexagonal networks. Surprisingly, we observed a very well-organized monolayer on the nematic film, characterized by a hexagonal organization similar to the one that can be obtained on a hard substrate (Supporting Figure S2 for comparison). The strong contrast associated with the AuNPs is consistent with the absence of nematic LC above the AuNPs, indeed already suggested by AFM results.

Localized Surface Plasmon Extinction. In order to understand if the AuNP organization is similar to that on a hard substrate, we have compared the localized surface plasmon (LSP) extinction of AuNPs directly deposited on top of the rubbed PVA substrate with that of the AuNPs organized in domains at the PSNLC surface (Figure 4). Figure 4 shows that for both systems a large red-shift of the LSP resonance wavelength is observed with respect to isolated AuNPs in solution (Supporting Figure S3). A similar red-shift is measured with NLC films with AuNPs (Supporting Figure S4). This shift reflects the electromagnetic coupling between NPs that occurs 
when they are packed within a hexagonal lattice. We have measured the LSP extinction for incident light parallel and perpendicular to the rubbing of PVA. Consistent with the symmetry of the hexagonal packing, we find no difference in the measurements and thus we only show nonpolarized data in Figure 4.

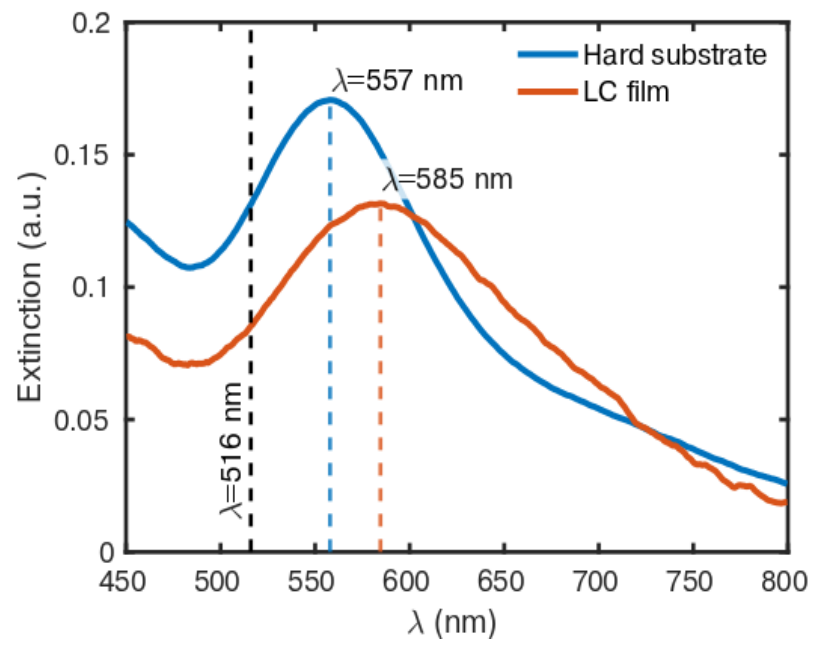

Figure 4. LSP extinction of AuNPs on a hard substrate (rubbed PVA) in blue, and on PSNLC

film in red. Maximum of each extinction curve is denoted by dashed lines. The LSP resonance of a AuNP toluene solution is indicated in the dashed black line.

A striking difference between the two data sets in Figure 4 is that the red-shift is significantly larger on top of the nematic film (69 $\mathrm{nm})$ as compared to the case on hard substrate without the LC (41 nm). It is known that for these NPs covered by ligands the optical index responsible for the LSP resonance wavelength is mostly imposed by the ligands. ${ }^{20,29}$ Thus the fact that $n_{e}=1.735$ is significantly larger than the optical index of PVA $(n=1.5)$ compared to $n_{o}=1.537$ is not at the origin of the observed larger red-shift for AuNPs at NLC surface. This is indeed confirmed by the 
observation that the LSP resonance does not depend on the polarization of the incident light. The difference of red-shift shown on Figure 4 thus leads to the following conclusion: On top of the nematic film there is an LC-induced shortening of the inter-NP distance, in other words a shortening of the period of the hexagonal network shown on Figure 3c with respect to that on a hard substrate without LC (Supporting Figure S2).

(a)

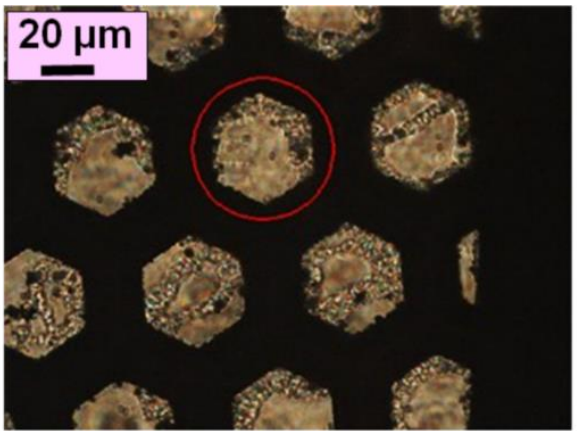

(c) $0 \mu \mathrm{m}$

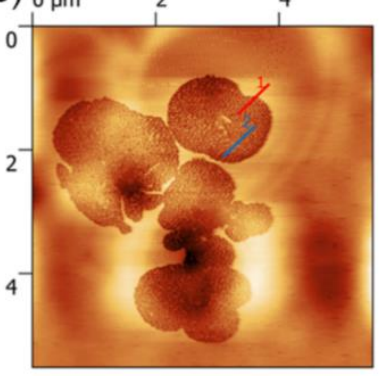

(d) $0 \mu \mathrm{m}$

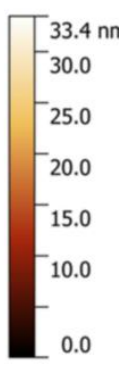

(b)
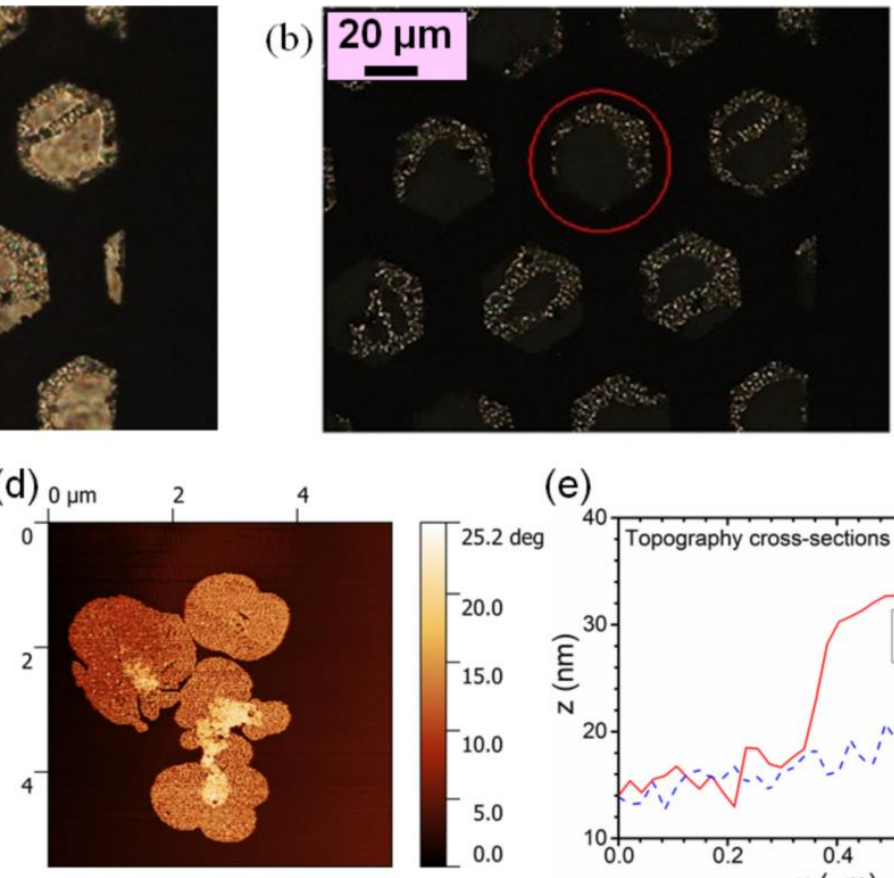

(e)

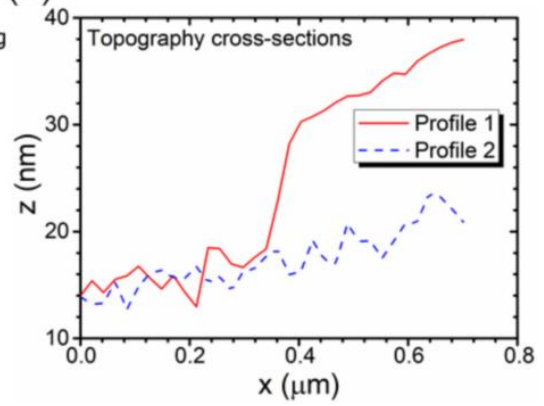

Figure 5. Optical microscopy images: (a) in transmission with parallel polarizer revealing the presence of LC film within the TEM copper grid, (b) with crossed polarizers, revealing a change of birefringence from homeotropic to tilted below the NPs. (c) AFM topographical and (d) phase data for a region with AuNPs domains on a 8CB smectic-A LC. (e) Corresponding topographical cross-sections according to the lines drawn on (c). AuNP regions are visible in the phase data by the variations of interaction with AFM tip which coincide with the hole areas observed in (c). The depth of the hole that the nanoparticles dig is around $15 \mathrm{~nm}$ with respect to the smectic surface at the hole edge. 


\section{DISCUSSION}

When the AuNP in toluene solution droplet is deposited on the nematic there is an almost complete dissolution of the nematic film. As the toluene evaporates, the nematic film reforms, leading to the AuNP localization at the air interface. This suggests that in absence of topological defects in the LC film, localization of AuNPs at the LC film surface may be the equilibrium state of the composite nematic film-AuNPs. Similar observations have been obtained for cholesteric films formed in the presence of platinum or gold nanoparticles, ${ }^{21,23}$ but, in this latter case, competing with formation of aggregates in the bulk..$^{22}$ Neither, in the NLC nor in the PSNLC film, aggregate is formed. This could be related to the preparation of the composites via the deposition of a droplet at the surface that somehow may favor localization at the air interface. In comparison with the cholesteric film, there is no modulation on the nematic surface. As a result no stripes of AuNPs are observed. ${ }^{21,23}$ Instead the AuNPs organize in a process that looks similar to the one leading to the well-known organization on top of a solid substrate after solvent evaporation (Supporting Figure S2). The filaments made of islands of ordered hexagonal AuNP networks revealed by combined POM, AFM and SEM may be connected to the end of solvent evaporation at the LC film surface. The islands are the signature of the last solvent droplets that bring AuNPs close to each other before full solvent evaporation. The observed NP organization is the usual result of inter-AuNP Van der Waals interactions that favor hexagonal organization of nanospheres. However spectrophotometric data demonstrate that on PSNLC (Figure 4) and on NLC (Supporting Figure S4) the period of the hexagonal network is shortened with respect to the one on a hard, rubbed PVA surface (without a LC). This suggests that an additional interaction takes place together with the inter-NP Van der Waals interactions. The inter-NP spacing in the hexagonal network has been measured on rubbed PVA surface (without a LC) by combined 
spectrophotometric measurements and synchrotron Grazing Incidence X-ray Scattering (GISAXS). It is equal to $1.9 \mathrm{~nm}^{20,30-32}$. If we analyze the red-shift of the plasmonic absorption of Figure 4 using the dipolar approximation (see "Materials and methods"), we find that the inter-NP gap decreases from $1.9 \mathrm{~nm}$ without $\mathrm{LC}^{20,30-32}$ to $1.1 \mathrm{~nm}$ on top of PSNLC, leading to a shortening of $0.8 \mathrm{~nm}$. The additional interaction thus leads to quite a large shortening of the inter-AuNP spacing that requires high distortion and interdigitation of the dodecanethiol ligands that have an extended length of $1.8 \mathrm{~nm}$. The nematic material allows for the formation of a well-organized AuNP network at the surface, however with a significantly enhanced electromagnetic coupling between AuNPs, able to significantly modify their optical properties. It is important to understand the origin of such shortening of lattice spacing. It may be related to the formation of the depression in the LC film below the AuNP domains. Since the observed shortening of the AuNP lattice spacing occurs independently of the polymerization in the nematic material (we find a similar redshift for plasmonic resonance with and without polymerization), this would imply that polymerization is not directly responsible for the establishment of the depressions. In order to check it and to understand the origin of the observed depressions, we have studied AuNP aggregation on smectic films without polymer.

Whereas it is very difficult to obtain reliable SEM or AFM pictures using nematic films without polymer, it is nonetheless possible to perform AFM measurements on smectic films. ${ }^{33}$ We prepared 8CB smectic films within TEM copper grids inducing homeotropic texture (see "Materials and Methods"), thus avoiding distortion and the creation of defects. ${ }^{34}$ After deposition of the same AuNP droplets on top of the smectic films, a similar anchoring modification of $8 \mathrm{CB}$ induced by the AuNPs is observed as revealed by POM (Figure 5a-b). Moreover, we observe the same localization of AuNPs at the $8 \mathrm{CB}$ surface, leading to the formation of similar depressions, as 
revealed by AFM measurements (Figure 5c-e). These results obtained with smectic films without polymerization confirm that polymerization in PSNLC, even if it impacts the depth of the depression, is not directly responsible for the formation of these domains at the LC surface nor for the formation of depression below AuNP domains at the surface of nematic or smectic films. They also suggest that the domains could correspond to the real equilibrium state of the composite nematic/smectic film-AuNPs, in agreement with the observations that the smectic films with AuNP domains at the surface remain unchanged several months after their preparation. Finally the fact that similar depression features below the AuNPs are observed in smectic and nematic films suggests that the origin of the depressions below AuNPs is similar in both phases. In the smectic phase the depressions can be viewed as the result of the tilted anchoring imposed below the AuNP domains: to allow for tilted anchoring and flat smectic layers, parallel to a flat interface, a (local) transition from a smectic-A phase to a smectic-C phase is necessary. However, along with the tilt comes layer compression, as in the smectic-A to smectic-C transition. The accommodation of compressed smectic-C layers and neighboring non-compressed smectic-A ones thus creates a depression below the AuNP domains (see scheme of Figure 6). For an anchoring tilt of $\theta$ below AuNP domains and a depression depth of about $h=15 \mathrm{~nm}$ corresponding to the one observed on Figure 5, the number of smectic layers involved in the smectic-C/smectic-A transition is larger than $N=(h+6) /\left(P^{*}(1-\cos (\theta))\right), 6 \mathrm{~nm}$ being the NP diameter, $P=3.16 \mathrm{~nm}$ being the smectic-A period of $8 \mathrm{CB}$. This leads to between 29 and 152 smectic layers involved in the smectic-C/smecticA transition for an anchoring tilt of $39^{\circ}$ and $17^{\circ}$ respectively. 


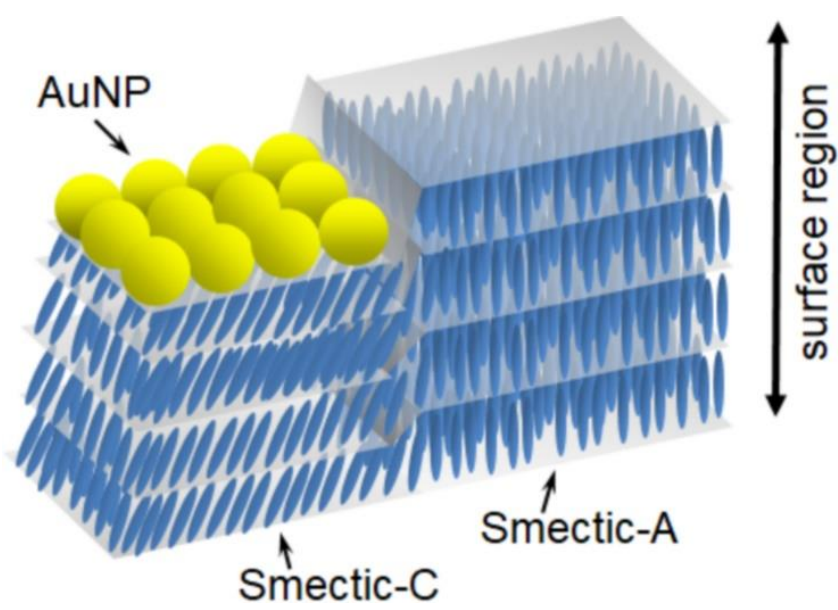

Figure 6. Schematic presentation of the depression occurring below AuNP domains due to a local smectic-C (below AuNP domain)/smectic-A (below air) transition.

How does this bear on our nematic data for 5CB? It has been observed in both experiment and modelling that nematics can display surface "freezing" and form several layers of smectic phase near a homeotropic surface. ${ }^{35,36}$ Since merely changing the anchoring condition from homeotropic to a tilt whose value ranges between $17^{\circ}$ and $39^{\circ}$ could not modify the nematic density, we postulate that a surface smectic-A phase forms and that, upon contact with the AuNPs, becomes a smectic-C. By analogy with the true equilibrium smectic of $8 \mathrm{CB}$, this should lead to the formation of a depression below AuNP domains. If we postulate that the polymerization has no influence on the depression feature, then the observed depression depth of $82 \mathrm{~nm}$ (the average value associated with more than 100 measurements) requires that the number of smectic- $\mathrm{A} / \mathrm{smectic}-\mathrm{C}$ layers to be very large, ranging from around 130 to 670 , as shown by the $\mathrm{N}$ values obtained for an anchoring tilt of $39^{\circ}$ and $17^{\circ}$, respectively, taking the $5 \mathrm{CB}$ smectic-A layer size close to the surface to be 2.6 nm. ${ }^{35}$ This number is definitely too large when compared to previous results suggesting that $5 \mathrm{CB}$ has short-range smectic order with at most 3 to 4 layers near the air interface ${ }^{35,36}$. This leads us to conclude that the polymerization must contribute to the observed large depression depth in 
PSNLC with AuNP monolayers. For instance, cybotactic clusters of polymers may co-organize with $5 \mathrm{CB}$ into layers close to the interface, with the polymer orientation being imposed by the $5 \mathrm{CB}$ thus inducing an increased layer size. ${ }^{37}$ The increased size of the polymer stabilized smectic layers would induce a large depth of the depressions below the AuNP domains. The polymerization may also favor the surface "freezing" of the nematic structure into a smectic one as previously shown in ref ${ }^{37}$ leading to a large number of polymer stabilized smectic layers close to the interface. Such a phenomenon may be enhanced by the directional UV-irradiation leading to the well-known favored polymerization close to the air-AuNP interface. ${ }^{38}$

The smectic-C structure induced by the tilted anchoring below AuNP domains incurs an energy penalty. To decrease this energy as much as possible the area of the AuNP domains will shrink, leading to the observed shortening of the lattice spacing. We can compare this shortening to prior work on AuNPs trapped in smectic topological defects. For small AuNPs, of diameter D $=4 \mathrm{~nm}$ with the same dodecanthiol ligands (with a size smaller than the core of the defects) no shortening is observed ${ }^{39}$. The AuNPs used here, of diameter $\mathrm{D}=6 \mathrm{~nm}$, have also been embedded in 2D smectic topological defects ${ }^{20,30}$. There, the $2 \mathrm{D}$ confinement induces formation of hexagonal networks similar to the one observed at the $5 \mathrm{CB}$ surface but instead embedded within the $2 \mathrm{D}$ defects. Shortening is also observed, suggesting that these AuNPs are larger than the defect core size in contrast with AuNPs of diameter $\mathrm{D}=4 \mathrm{~nm}$. The shortening is only $0.6 \mathrm{~nm}$, of the same order yet smaller than that observed here on 5CB (with both PSNLC and NLC film). In the former case the advantage of being embedded within a topological defect core may be responsible of the smaller shortening. This however shows that the disorder induced by the AuNPs outside of the topological defect core has an energy per unit area of the same order of magnitude as the one associated with the putative smectic-C/smectic-A induced by the AuNPs. 
These results finally show that the LC anchoring modifications induced by the AuNP domains allow to manipulate the AuNP domain lattice spacing and to enhance the coupling between AuNPs. For metallic NPs this induces a significant red-shift of the plasmonic resonance known to be associated with an enhanced exaltation of the electromagnetic field between neighboring NPs under light irradiation. This will be particularly appropriate to use these composite surfaces as Surface Enhanced Raman Scattering (SERS) surfaces, for sensing applications. This effect could even be monitored using different ligands around AuNPs to further modify the LC anchoring below AuNP domains and thus still enhancing the coupling between NPs. It will be also interesting to study the possible activation of plasmonic resonance as a function of temperature, the temperature being increased above the nematic/isotropic transition $\left(35.2^{\circ} \mathrm{C}\right.$ for $\left.5 \mathrm{CB}\right)$ where the $\mathrm{LC}$ anchoring phenomenon is lost. Increase of temperature above the transition may eliminate the lattice spacing shortening. Temperature may thus become an external activator for control of the coupling between NPs in ordered NP networks when they are formed on top of nematic or smectic surfaces.

\section{CONCLUSION}

In conclusion, we have shown that nematic films can serve as substrates for the formation of wellordered hexagonal monolayers of gold nanoparticles (AuNPs) of diameter $\mathrm{D}=6 \mathrm{~nm}$. This has been shown by combining Optical Microscopy on Polymerized Stabilized 5CB (PSNLC) and pure 5CB (NLC) films, Atomic Force Microscopy (AFM), Scanning Electron microscopy (SEM) on PSNLC films, and Spectrophotometry on PSNLC films and NLC films. The same features have been shown on smectic surfaces by combining Optical Microscopy and AFM. However we also show how both AuNP networks and nematic films are mutually impacted by each other. The AuNP network period is shortened by the surrounding nematic film and depressions are created at the 
nematic surface, where the AuNP domains are formed. We show that it is related to a tilt of the nematic anchoring induced below the AuNP monolayers, ranging from $17^{\circ}$ to $39^{\circ}$. In the smectic film the anchoring tilt transforms smectic-A layers into smectic-C layers. Depressions through layer-shrinkage may then become necessary to accommodate the neighboring smectic-A (without AuNPs) and smectic-C (with AuNP monolayers) areas. The same phenomenon occurs with nematic films in relation with the known surface-induced smectic-A structure of the nematic film at the interface transformed into a smectic-C one. The shortening of the AuNP network period may be induced by the necessary minimization of the surface of the smectic-C areas in relation to the energy cost of the local smectic-A/smectic-C transition. We find that when the nematic film is polymer stabilized the depression depth is increased either in relation with an increased smectic layer size or with an increased number of polymer stabilized smectic layers close to the surface with respect to NLC films. These results not only open the route for a control of the coupling between NPs at LC surface through the use of various ligands around NPs that would modify the LC anchoring underneath the AuNP domains, but also for activated control through temperature changes reaching the nematic/smectic transition temperature.

\section{MATERIALS AND METHODS}

Solid substrate preparation. PVA was spin-coated onto clean glass slides, baked for at least one hour at $120^{\circ} \mathrm{C}$ to polymerize, and rubbed unidirectionally with a commercial rubbing cloth to promote planar anchoring at the surface. This treatment is used on the silicon (SI) substrate to visualize the AuNPs without the LC, on glass slides for NLC and PSNLC samples, and on those coated with a thin transparent layer of Indium Thin Oxide (ITO) to visualize PSNLC samples by SEM. 
Photopolymerizable Monomer-Nematic LC Mixture. In order to obtain PSNLC films, 4-pentyl4'-cyanobiphenyl (5CB, Sigma-Aldrich - Figure 7a) was mixed with RM257 (30 wt\%, Synthon Chemicals GmbH \& Co. KG - Figure 7b) and a photo-initiator (1Hydroxycyclohexylphenylketone, 1,2 wt $\%$, Sigma-Aldrich). To ensure better miscibility, dicholoromethane is added. The LC monomer mixture is heated in a closed flask at $60^{\circ} \mathrm{C}$ for 45 minutes, during which the dicholormethane is in the gaseous phase. Once homogeneous, the flask is opened and all dichloromethane evaporates. 
(a) 4-pentyl-4-biphenylcarbonitrile: $\mathrm{Cr} \stackrel{22^{\circ} \mathrm{C}}{\leftrightarrow} \mathrm{N} \stackrel{35.2^{\circ} \mathrm{C}}{\leftrightarrow} \mathrm{I}$

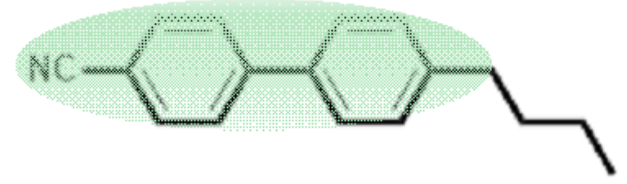

(b) RM257:

$\mathrm{Cr} \stackrel{70^{\circ} \mathrm{C}}{\leftrightarrow} \mathrm{N} \stackrel{126^{\circ} \mathrm{C}}{\leftrightarrow}$ I (d) Dodecanethiols stabilized Au

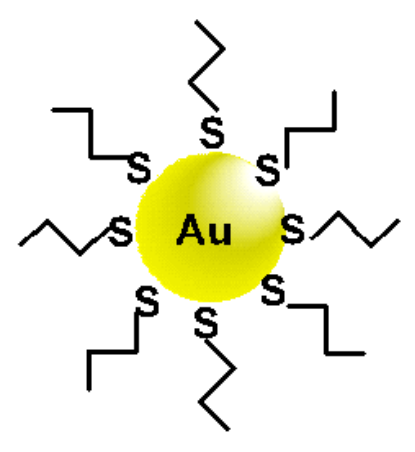

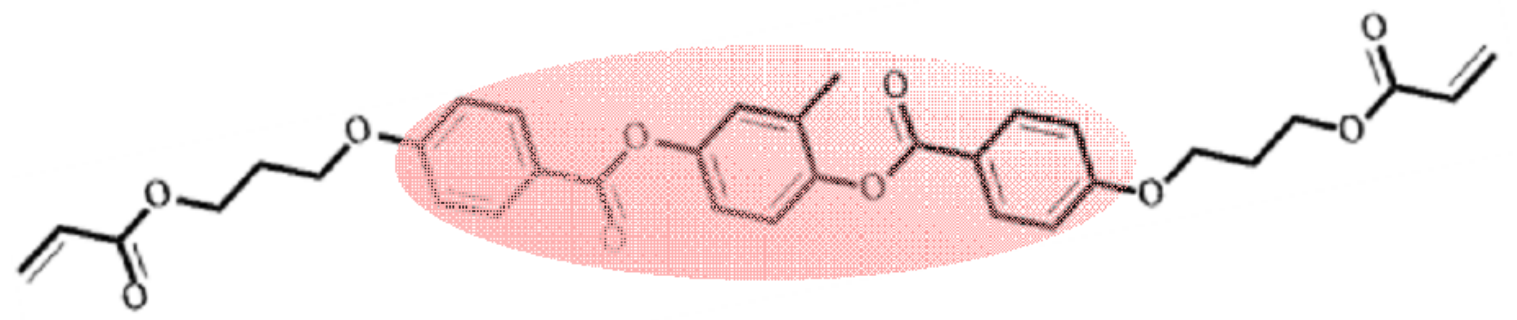

(c)

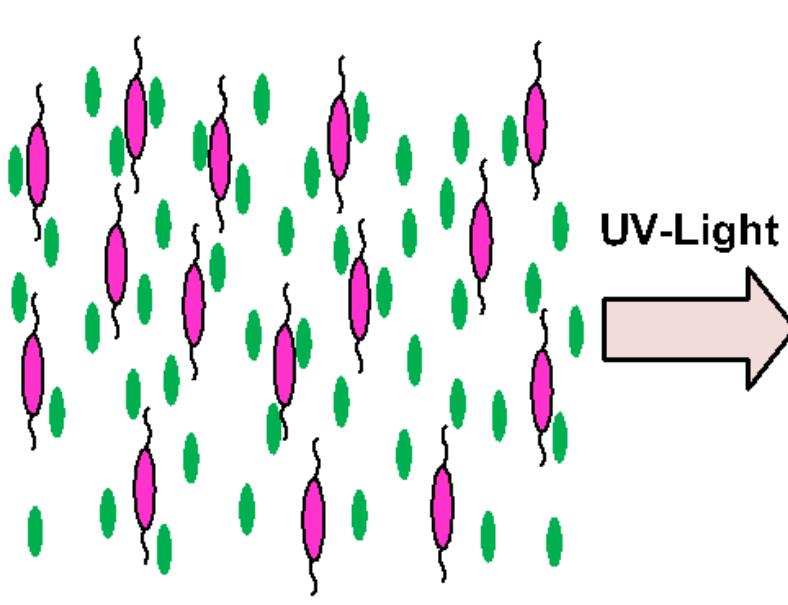

Before polymerization

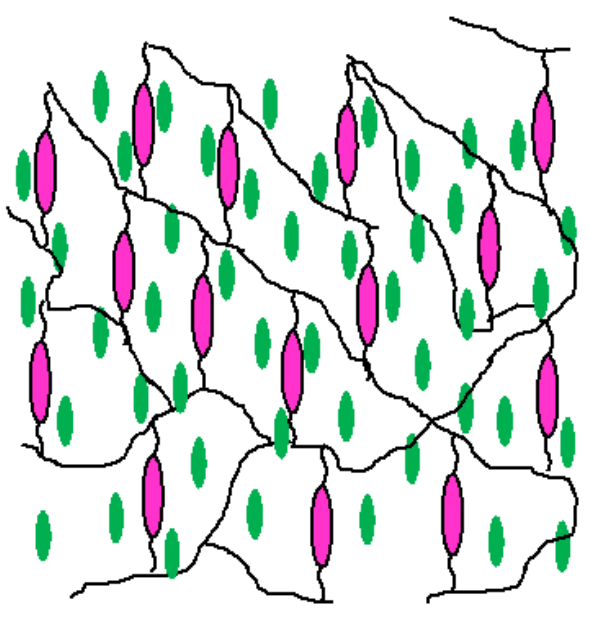

After polymerization

Figure 7. Chemical structures of (a) 5CB and (b) RM257. (c) Schematic presentation of the polymerization by UV irradiation of the reactive monomers RM257 to form PSNLC film. RM257 are represented in pink; 5CB are represented in green. (d) AuNP of diameter $6 \mathrm{~nm}$. 
AuNP Synthesis. 1, 2, 3, 4-tetrahydronaphthalene (tetralin, 99\% Aldrich), Chlorotriphenylphosphine $\mathrm{Au}(\mathrm{I})$ (98\%) and tert-butylamine borane (97\%) were obtained from STEM chemicals. Dodecanethiol (DDT), oleylamine, HAuCl4 (98\%), hexane, were obtained from Sigma-Aldrich, Toluene (98\%) from Riedel de Haen. Ethanol (99.85\%), chloroform (99.2\%) from VWR. All reagents were used as received without further purification. The synthesis is based on reference. ${ }^{40} 50 \mathrm{mg}$ of $\mathrm{HauCl} 4$ are mixed in a three neck flask with $5 \mathrm{~mL}$ of oleylamine and $5 \mathrm{~mL}$ of tetralin and degassed at room temperature. The flask is dipped in an ice bath. The temperature controller is put in the ice bath rather than in the three neck flask. Meanwhile, $22 \mathrm{mg}$ of tertbutylborane is mixed with $0.5 \mathrm{~mL}$ of oleylamine and $0.5 \mathrm{~mL}$ of tetratlin. The mixture is sonicated until full dissolution of the salt. The three neck flask is put under Ar. The borane solution is injected promptly. The solution changes color to brown and then to purple. Reaction is continued for $36 \mathrm{~min} .0 .5 \mathrm{~mL}$ of DDT is added to stop the reaction growth. The content of the flask is mixed with $5 \mathrm{~mL}$ of ethanol and then centrifuged. The formed pellet is redispersed in toluene. NPs of diameter $\mathrm{D}=6 \mathrm{~nm}$ surrounded by dodecanethiol ligands (Figure $7 \mathrm{~d}$ ) and polydispersity 9\% were obtained as shown by SAXS measurements performed on synchrotron Soleil.

Nematic LC and PSNLC film preparation with or without AuNPs. Once the PVA-coated glass is prepared, $10 \mu \mathrm{l}$ of the nematic material is spin-coated (2000 rpm for $20 \mathrm{~s}$ ) to achieve uniform thickness throughout the film. This effectively generates a hybrid film, with planar anchoring on the PVA substrate, and homeotropic anchoring at the nematic-air interface. The uniformity in thickness is checked by optical microscopy and Profilometer measurements. The AuNPs solution (with a concentration of $5.6 \times 10^{17} \mathrm{AuNPs} / \mathrm{L}$ ) is deposited by drop casting on the nematic film. Evaporation of the solvent can either induce contraction of the LC film or division into two or three smaller films, depending on the solvent evaporation. To induce polymerization 
(Figure 7c), the samples were irradiated in a nitrogen atmosphere with UV light of $0.15 \mathrm{~mW} . \mathrm{cm}^{-2}$ intensity at wavelength $365 \mathrm{~nm}$ for $20 \mathrm{~min}$ at room temperature. ${ }^{41,42}$

Smectic LC Film Preparation with AuNPs. Homeotropic substrates were prepared by spincoating $50 \mu \mathrm{l}$ of a $0.1 \mathrm{wt} \%$ DMOAP solution on clean glass and were baked at $100{ }^{\circ} \mathrm{C}$ for 1 hour. Hexagonal $\mathrm{Cu}$ grids were placed on the substrate to ensure uniform thickness and that a uniform homeotropic smectic film is formed. ${ }^{34}$ The LC, in this case $8 \mathrm{CB}$, purchased from Sigma-Aldrich, was heated above its clearing temperature and deposited on the grids. The film cools down and at room temperature forms a smectic phase. Optical microscopy images were taken to confirm a homeotropic film was formed. To deposit AuNPs on the smectic film, a $10 \mu 1$ solution of AuNPs and toluene is deposited $1 \mu \mathrm{l}$ at a time, and enough time is given to allow the toluene to evaporate.

\section{Samples Characterization:}

POM: The manufactured films are visualized by a LEICA DMRX microscope, surmounted by a SONY CCD camera. A Xenon lamp illuminates the sample in white light, placed so that the rubbing direction is oriented at $45^{\circ}$ from the polarizer and the analyzer. This position allows us to have the maximum intensity.

AFM: AFM images were obtained on PSNLC films and on smectic films with and without AuNPs using a Veeco model Nanoscope Dimension 3100 Atomic Force Microscope and a $500 \mathrm{kHz}$ cantilever/stylus in tapping mode.

SEM: SEM images were obtained on PSNLC films with AuNPs using a ZEISS scanning electron microscope. 
Spectrophotometry: The extinction spectra for spherical isolated AuNPs in toluene was measured using a Cary 5000 UV-Vis-NIR. The experimental data were fitted with a Gaussian curve. Spectra for the AuNPs of $6 \mathrm{~nm}$ in diameter display a plasmon resonance of $516 \mathrm{~nm}$.

A Maya 2000 Pro micro spectrophotometer coupled to the Leica optical microscope through optical fiber was used to measure the light absorption of PSNLC-AuNP and NLC samples. The sample was illuminated by a white light source, and areas of $150 \mu \mathrm{m} \times 150 \mu \mathrm{m}$ are probed with a 50x objective lens. All spectra were taken at room temperature in the 190-1100 nm spectral range. A reference made of PSNLC (or NLC) of similar thickness but without AuNP was used to extract the optical absorption of AuNPs only.

The distance between AuNPs was extracted from the extinction spectra using a dipole coupling model in the quasistatic approximation (NPs diameter D « wavelength $\lambda$ ). ${ }^{43}$ In this approximation the multipolar interactions between AuNPs as well as the retardance effect are not considered, this latter assumption being obviously correct due the small size of AuNPs. In this case the resonance condition can be calculated using the expression of the effective polarizability of an infinite AuNP hexagonal monolayer:

$\alpha_{\text {eff }}=\alpha /\left(1-\alpha \Sigma / 4 \pi(D+s)^{3}\right)$

with $\alpha=4 \pi(\mathrm{D} / 2)^{3}\left(\varepsilon-\varepsilon_{\mathrm{m}}\right) /\left(\varepsilon+2 \varepsilon_{\mathrm{m}}\right)$ is the polarizability of a single AuNP, leading for the plasmonic resonance to the following equation:

$$
\varepsilon=\varepsilon_{\mathrm{m}}\left(\Sigma+8(\mathrm{~s} / \mathrm{D}+1)^{3}\right) /\left(\Sigma-4(\mathrm{~s} / \mathrm{D}+1)^{3}\right)^{43}
$$

that relates $\varepsilon$ the AuNP dielectric function, $\varepsilon_{\mathrm{m}}$ the dielectric function of the homogeneous surrounding, $\mathrm{s}$ the inter-AuNP gap in the hexagonal network and $\mathrm{D}$ the AuNP diameter $(\mathrm{D}=6$ 
$\mathrm{nm}) . \Sigma$ is the lattice sum associated with the AuNPs assembly, AuNPs being considered as periodic dipoles. For monolayers considered as an infinite hexagonal network, the sum $\Sigma$ is $\Sigma \approx 5.5$. 44,45

The AuNP dielectric function was recalculated based on Johnson and Christy data ${ }^{46}$ to take into account the small size effect and the influence of the chemical interface, leading to broadening and blue-shifted localized surface plasmon resonance of the isolated NPs in toluene. ${ }^{47} \varepsilon_{\mathrm{m}}$ is taken as equal to 2.28 , previously shown to be mostly induced by the dodecanethiol ligands for these same AuNPs. $^{20}$

\section{ASSOCIATED CONTENT}

Supporting Information. The Supporting Information is available free of charge on the ACS Publications website at DOI:

Optical microscopy image of nematic liquid crystal film in the presence of AuNPs, SEM image of a self-organized AuNPs on unidirectionally rubbed PVA on a silicon substrate, LSP extinction of isolated AuNPs suspended in toluene and of AuNPs on nematic film (PDF)

\section{AUTHOR INFORMATION}

\section{Corresponding Author}

*E-mail: emmanuelle.lacaze@insp.jussieu.fr

\section{Notes}

The authors declare no competing financial interest.

\section{ACKNOWLEDGMENT}


We would like to thank Abdelhafidh Gharbi for precious comments and fruitful discussions, Christophe Blanc for useful help with the polymerization test, Denis Limagne for technical supports. Ines Gharbi was supported by the Tunisian Ministry for Higher Education, Research, and Technology and by the CNRS-UMR7588. Ines Gharbi's travel between Tunis and Paris was supported by a grant from Université de Tunis El Manar and more specifically from LR99ES16. Viviana Palacio-Betancur is thankful to the Fulbright commission in Colombia and Colciencias, as well as the Chateaubriand Fellowship program and the Eiffel Excellence Scholarship program. Randall D. Kamien was supported by NSF MRSEC Grant DMR-1720530 and by a Simons Investigator Grant from the Simons Foundation. We are grateful to Brigitte Pansu for providing Au nanoparticles and thank Soleil synchrotron and Doru Constantin for the measurement of the diameter and polydispersity in size of AuNPs using SAXS on the beamline Swing.

\section{REFERENCES AND NOTES}

(1) Blanc, C.; Coursault, D.; Lacaze, E. Ordering Nano- and Microparticles Assemblies with Liquid Crystals. Liquid Crystals Reviews 2013, 1 (2), 83-109. https://doi.org/10.1080/21680396.2013.818515.

(2) Lagerwall, J. P. F.; Scalia, G. Liquid Crystals with Nano and Microparticles. 2017, 26 (4), 126-127. https://doi.org/10.1080/1358314X.2017.1401269.

(3) Choudhary, A.; Singh, G.; Biradar, A. M. Advances in Gold Nanoparticle-Liquid Crystal Composites. Nanoscale 2014, 6 (14), 7743-7756. https://doi.org/10.1039/C4NR01325E.

(4) Sridevi, S.; Prasad, S. K.; Nair, G. G.; D’Britto, V.; Prasad, B. L. V. Enhancement of Anisotropic Conductivity, Elastic, and Dielectric Constants in a Liquid Crystal-Gold Nanorod System. Appl. Phys. Lett. 2010, $97 \quad$ (15), 151913. https://doi.org/10.1063/1.3499744.

(5) Mishra, M.; Kumar, S.; Dhar, R. Effect of Dispersed Colloidal Gold Nanoparticles on the Electrical Properties of a Columnar Discotic Liquid Crystal. RSC Adv. 2014, 4 (107), 62404-62412. https://doi.org/10.1039/C4RA11541D.

(6) Tomašovičová, N.; Timko, M.; Mitróová, Z.; Koneracká, M.; Rajňak, M.; Éber, N.; TóthKatona, T.; Chaud, X.; Jadzyn, J.; Kopčanský, P. Capacitance Changes in Ferronematic Liquid Crystals Induced by Low Magnetic Fields. Phys. Rev. E 2013, 87 (1), 014501. https://doi.org/10.1103/PhysRevE.87.014501.

(7) Mishra, R.; Hazarika, J.; Hazarika, A.; Gogoi, B.; Dubey, R.; Bhattacharjee, D.; Singh, K. N.; Alapati, P. R. Dielectric Properties of a Strongly Polar Nematic Liquid Crystal Compound Doped with Gold Nanoparticles. Liquid Crystals 2018, 45 (11), 1661-1671. https://doi.org/10.1080/02678292.2018.1478995. 
(8) Lee, E.; Xia, Y.; Ferrier, R. C.; Kim, H.-N.; Gharbi, M. A.; Stebe, K. J.; Kamien, R. D.; Composto, R. J.; Yang, S. Fine Golden Rings: Tunable Surface Plasmon Resonance from Assembled Nanorods in Topological Defects of Liquid Crystals. Advanced Materials 2016, 28 (14), 2731-2736. https://doi.org/10.1002/adma.201506084.

(9) Qi, H.; Hegmann, T. Formation of Periodic Stripe Patterns in Nematic Liquid Crystals Doped with Functionalized Gold Nanoparticles. J. Mater. Chem. 2006, 16 (43), 4197-4205. https://doi.org/10.1039/B611501B.

(10) Qi, H.; Kinkead, B.; Marx, V. M.; Zhang, H. R.; Hegmann, T. Miscibility and Alignment Effects of Mixed Monolayer Cyanobiphenyl Liquid-Crystal-Capped Gold Nanoparticles in Nematic Cyanobiphenyl Liquid Crystal Hosts. ChemPhysChem 2009, 10 (8), 1211-1218. https://doi.org/10.1002/cphc.200800765.

(11) Yoshida, H.; Tanaka, Y.; Kawamoto, K.; Kubo, H.; Tsuda, T.; Fujii, A.; Kuwabata, S.; Kikuchi, H.; Ozaki, M. Nanoparticle-Stabilized Cholesteric Blue Phases. Applied Physics Express 2009, 2 (12), 121501. https://doi.org/10.1143/APEX.2.121501.

(12) Karatairi, E.; Rožič, B.; Kutnjak, Z.; Tzitzios, V.; Nounesis, G.; Cordoyiannis, G.; Thoen, J.; Glorieux, C.; Kralj, S. Nanoparticle-Induced Widening of the Temperature Range of Liquid-Crystalline Blue Phases. Phys. Rev. E 2010, 81 (4), 041703. https://doi.org/10.1103/PhysRevE.81.041703.

(13) Rožič, B.; Tzitzios, V.; Karatairi, E.; Tkalec, U.; Nounesis, G.; Kutnjak, Z.; Cordoyiannis, G.; Rosso, R.; Virga, E. G.; Muševič, I.; Kralj, S. Theoretical and Experimental Study of the Nanoparticle-Driven Blue Phase Stabilisation. Eur. Phys. J. E 2011, 34 (2), 17. https://doi.org/10.1140/epje/i2011-11017-8.

(14) Senyuk, B.; Evans, J. S.; Ackerman, P. J.; Lee, T.; Manna, P.; Vigderman, L.; Zubarev, E. R.; van de Lagemaat, J.; Smalyukh, I. I. Shape-Dependent Oriented Trapping and Scaffolding of Plasmonic Nanoparticles by Topological Defects for Self-Assembly of Colloidal Dimers in Liquid Crystals. Nano Lett. 2012, 12 (2), 955-963. https://doi.org/10.1021/nl204030t.

(15) Cordoyiannis, G.; Jampani, V. S. R.; Kralj, S.; Dhara, S.; Tzitzios, V.; Basina, G.; Nounesis, G.; Kutnjak, Z.; Tripathi, C. S. P.; Losada-Pérez, P.; Jesenek, D.; Glorieux, C.; Muševič, I.; Zidanšek, A.; Ameinitsch, H.; Thoen, J. Different Modulated Structures of Topological Defects Stabilized by Adaptive Targeting Nanoparticles. Soft Matter 2013, 9 (15), 39563964. https://doi.org/10.1039/C3SM27644A.

(16) Evans, J. S.; Ackerman, P. J.; Broer, D. J.; J. Lagemaat, V. D.; Smalyukh, I. I. Optical Generation, Templating, and Polymerization of Three-Dimensional Arrays of LiquidCrystal Defects Decorated by Plasmonic Nanoparticles. Physical Review E 2013, 87 (3), 032503-1/14. https://doi.org/10.1103/PhysRevE.87.032503.

(17) Gharbi, M. A.; Manet, S.; Lhermitte, J.; Brown, S.; Milette, J.; Toader, V.; Sutton, M.; Reven, L. Reversible Nanoparticle Cubic Lattices in Blue Phase Liquid Crystals. ACS Nano 2016, 10 (3), 3410-3415. https://doi.org/10.1021/acsnano.5b07379.

(18) Rožič, B.; Fresnais, J.; Molinaro, C.; Calixte, J.; Umadevi, S.; Lau-Truong, S.; Felidj, N.; Kraus, T.; Charra, F.; Dupuis, V.; Hegmann, T.; Fiorini-Debuisschert, C.; Gallas, B.; Lacaze, E. Oriented Gold Nanorods and Gold Nanorod Chains within Smectic Liquid Crystal Topological Defects. ACS Nano 2017, 11 (7), 6728-6738. https://doi.org/10.1021/acsnano.7b01132. 
(19) Mundoor, H.; Sheetah, G. H.; Park, S.; Ackerman, P. J.; Smalyukh, I. I.; van de Lagemaat, J. Tuning and Switching a Plasmonic Quantum Dot "Sandwich" in a Nematic Line Defect. ACS Nano 2018, 12 (3), 2580-2590. https://doi.org/10.1021/acsnano.7b08462.

(20) Do, S.-P.; Missaoui, A.; Coati, A.; Resta, A.; Goubet, N.; Royer, S.; Guida, G.; Briand, E.; Lhuillier, E.; Garreau, Y.; Babonneau, D.; Goldmann, M.; Constantin, D.; Croset, B.; Gallas, B.; Lacaze, E. Interactions Between Topological Defects and Nanoparticles. Front. Phys. 2020, 7, 234. https://doi.org/10.3389/fphy.2019.00234.

(21) Mitov, M.; Portet, C.; Bourgerette, C.; Snoeck, E.; Verelst, M. Long-Range Structuring of Nanoparticles by Mimicry of a Cholesteric Liquid Crystal. Nat Mater 2002, 1 (4), 229-231. https://doi.org/10.1038/nmat772.

(22) Mitov, M.; Bourgerette, C.; Guerville, F. de. Fingerprint Patterning of Solid Nanoparticles Embedded in a Cholesteric Liquid Crystal. J. Phys.: Condens. Matter 2004, 16 (19), S1981S1988. https://doi.org/10.1088/0953-8984/16/19/010.

(23) Bitar, R.; Agez, G.; Mitov, M. Cholesteric Liquid Crystal Self-Organization of Gold Nanoparticles. Soft Matter 2011, 7 (18), 8198-8206. https://doi.org/10.1039/C1SM05628J.

(24) Tran, L.; Kim, H.-N.; Li, N.; Yang, S.; Stebe, K. J.; Kamien, R. D.; Haase, M. F. Shaping Nanoparticle Fingerprints at the Interface of Cholesteric Droplets. Science Advances 2018, 4 (10), eaat8597. https://doi.org/10.1126/sciadv.aat8597.

(25) Miller, D. S.; Carlton, R. J.; Mushenheim, P. C.; Abbott, N. L. Introduction to Optical Methods for Characterizing Liquid Crystals at Interfaces. Langmuir 2013, 29 (10), 3154 3169. https://doi.org/10.1021/la304679f.

(26) Lelidis, I.; Gharbi, A.; Durand, G. A New Optical Method to Measure Angular Tilts for Planar Anchored Nematic Liquid Crystals. Molecular Crystals and Liquid Crystals Science and Technology. Section A. Molecular Crystals and Liquid Crystals 1992, 223 (1), 263276. https://doi.org/10.1080/15421409208048257.

(27) Mohammadimasoudi, M.; Neyts, K.; Beeckman, J. Thin Film Polarizer and Color Filter Based on Photo-Polymerizable Nematic Liquid Crystal. In Emerging Liquid Crystal Technologies X; International Society for Optics and Photonics, 2015; Vol. 9384, p 93840E. https://doi.org/10.1117/12.2079516.

(28) Drawhorn, R. A.; Abbott, N. L. Anchoring of Nematic Liquid Crystals on Self-Assembled Monolayers Formed from Alkanethiols on Semitransparent Films of Gold. J. Phys. Chem. 1995, 99 (45), 16511-16515. https://doi.org/10.1021/j100045a004.

(29) Mulvaney, P. Surface Plasmon Spectroscopy of Nanosized Metal Particles. Langmuir 1996, 12 (3), 788-800. https://doi.org/10.1021/la9502711.

(30) Do, S.-P.; Missaoui, A.; Coati, A.; Coursault, D.; Jeridi, H.; Resta, A.; Goubet, N.; Wojcik, M. M.; Choux, A.; Royer, S.; Briand, E.; Donnio, B.; Gallani, J. L.; Pansu, B.; Lhuillier, E.; Garreau, Y.; Babonneau, D.; Goldmann, M.; Constantin, D.; Gallas, B.; Croset, B.; Lacaze, E. From Chains to Monolayers: Nanoparticle Assembly Driven by Smectic Topological Defects. Nano Lett. 2020, 20 (3), 1598-1606. https://doi.org/10.1021/acs.nanolett.9b04347.

(31) Goubet, N.; Richardi, J.; Albouy, P.-A.; Pileni, M.-P. Which Forces Control Supracrystal Nucleation in Organic Media? Advanced Functional Materials 2011, 21 (14), 2693-2704. https://doi.org/10.1002/adfm.201100382.

(32) Gauvin, M.; Wan, Y.; Arfaoui, I.; Pileni, M.-P. Mechanical Properties of Au Supracrystals Tuned by Flexible Ligand Interactions. J. Phys. Chem. C 2014, 118 (9), 5005-5012. https://doi.org/10.1021/jp411896c. 
(33) Michel, J.-P.; Lacaze, E.; Alba, M.; De Boissieu, M.; Gailhanou, M.; Goldmann, M. Optical Gratings Formed in Thin Smectic Films Frustrated on a Single Crystalline Substrate. Phys Rev E Stat Nonlin Soft Matter Phys 2004, 70 (1 10 Pt 1), 011709. https://doi.org/10.1103/PhysRevE.70.011709.

(34) Brake, J. M; Abbott, N. L. An Experimental System for Imaging the Reversible Adsorption of Amphiphiles at Aqueous-Liquid Crystal Interfaces. Langmuir 2002, 18 (16), 6101-6109. https://doi.org/10.1021/la011746t.

(35) Fukuto, M.; Gang, O.; Alvine, K. J.; Ocko, B. M.; Pershan, P. S. Wetting of Liquid-Crystal Surfaces and Induced Smectic Layering at a Nematic-Liquid Interface: An x-Ray Reflectivity Study. Phys Rev E Stat Nonlin Soft Matter Phys 2008, 77 (3 Pt 1), 031607. https://doi.org/10.1103/PhysRevE.77.031607.

(36) Sadati, M.; Ramezani-Dakhel, H.; Bu, W.; Sevgen, E.; Liang, Z.; Erol, C.; Rahimi, M.; Taheri Qazvini, N.; Lin, B.; Abbott, N. L.; Roux, B.; Schlossman, M. L.; de Pablo, J. J. Molecular Structure of Canonical Liquid Crystal Interfaces. J. Am. Chem. Soc. 2017, 139 (10), 3841-3850. https://doi.org/10.1021/jacs.7b00167.

(37) Noh, J.; Henx, B.; Lagerwall, J. P. F. Taming Liquid Crystal Self-Assembly: The Multifaceted Response of Nematic and Smectic Shells to Polymerization. Advanced Materials 2016, 28 (46), 10170-10174. https://doi.org/10.1002/adma.201603158.

(38) Kamal, T.; Park, S.-Y. A Liquid Crystal Polymer Based Single Layer Chemo-Responsive Actuator. Chem. Commun. 2014, $50 \quad$ (16), 2030-2033. https://doi.org/10.1039/C3CC48409B.

(39) Coursault, D.; Blach, J.-F.; Grand, J.; Coati, A.; Vlad, A.; Zappone, B.; Babonneau, D.; Lévi, G.; Félidj, N.; Donnio, B.; Gallani, J.-L.; Alba, M.; Garreau, Y.; Borensztein, Y.; Goldmann, M.; Lacaze, E. Tailoring Anisotropic Interactions between Soft Nanospheres Using Dense Arrays of Smectic Liquid Crystal Edge Dislocations. ACS Nano 2015, 9 (12), 11678-11689. https://doi.org/10.1021/acsnano.5b02538.

(40) Peng, S.; Lee, Y.; Wang, C.; Yin, H.; Dai, S.; Sun, S. A Facile Synthesis of Monodisperse $\mathrm{Au}$ Nanoparticles and Their Catalysis of CO Oxidation. Nano Res. 2008, 1 (3), 229-234. https://doi.org/10.1007/s12274-008-8026-3.

(41) Dierking, I. Polymer Network-Stabilized Liquid Crystals. Advanced Materials 2000, 12 (3), 167-181. https://doi.org/10.1002/(SICI)1521-4095(200002)12:3<167::AIDADMA167>3.0.CO;2-I.

(42) Gharbi, I.; Missaoui, A.; Demaille, D.; Lacaze, E.; Rosenblatt, C. Persistence of Smectic-A Oily Streaks into the Nematic Phase by UV Irradiation of Reactive Mesogens. Crystals 2017, 7 (12), 358. https://doi.org/10.3390/cryst7120358.

(43) Kreibig, U.; Vollmer, M. Optical properties of metal clusters.

(44) Topping, J.; Chapman, S. On the Mutual Potential Energy of a Plane Network of Doublets. Proceedings of the Royal Society of London. Series A, Containing Papers of a Mathematical and Physical Character 1927, 114 (766), 67-72. https://doi.org/10.1098/rspa.1927.0025.

(45) van der Hoff, B. M. E.; Benson, G. C. A Method for the Evaluation of Some Lattice Sums Occurring in Calculations of Physical Properties of Crystals. Can. J. Phys. 1953, 31 (7), 1087-1094. https://doi.org/10.1139/p53-093.

(46) Johnson, P. B.; Christy, R. W. Optical Constants of the Noble Metals. Phys. Rev. B 1972, 6 (12), 4370-4379. https://doi.org/10.1103/PhysRevB.6.4370. 
(47) Garcia, M. A.; de la Venta, J.; Crespo, P.; LLopis, J.; Penadés, S.; Fernández, A.; Hernando, A. Surface Plasmon Resonance of Capped Au Nanoparticles. Phys. Rev. B 2005, 72 (24), 241403. https://doi.org/10.1103/PhysRevB.72.241403. 